\title{
Management of Recurrent Nasal Dermoid in an Adolescent with Nasal Pyramid Deformity
}

\author{
Dasari Samuel Deenadayal, Bashettynaveen Kumar, Ramesh Chinta, Vyshanavi Bommakanti, \\ Ramesh Chinta
}

Deenadayal ENT Care Centre, Secunderabad, India

Email: vyshnavi.jajee@gmail.com

How to cite this paper: Deenadayal, D.S., Kumar, B., Chinta, R., Bommakanti, V. and Chinta, R. (2018) Management of Recurrent Nasal Dermoid in an Adolescent with Nasal Pyramid Deformity. International Journal of Otolaryngology and Head \& Neck Surgery, 7, 132-137.

https://doi.org/10.4236/ijohns.2018.73015

Received: February 7, 2018

Accepted: May 27, 2018

Published: May 30, 2018

Copyright $\odot 2018$ by authors and Scientific Research Publishing Inc. This work is licensed under the Creative Commons Attribution International License (CC BY 4.0).

http://creativecommons.org/licenses/by/4.0/

\begin{abstract}
Nasal dermoids are congenital midline masses accounting for $1 \%-3 \%$ of all dermoids. The majority of lesions are superficial with no deep extension; a variable reported percentage might end blindly within the deeper midline structures of the nose or extend intracranially. Sequelae of infection can result in osteomyelitis, meningitis or cerebral abscess. These complications may result from inadequate surgical excision. We report a case of recurrent nasal dermoid that had associated nasal pyramid deformity.
\end{abstract}

\section{Keywords}

Nasal Dermoids, Navigation

\section{Introduction}

A variety of congenital midline nasal masses occurs in children. These disorders are clinically important because of their potential connection to the central nervous system. Congenital midline nasal masses include nasal dermoids, nasal gliomas, and encephaloceles. These are relatively rare congenital anomalies, which are estimated to occur in 1 in 20,000 - 40,000 births [1]. Nasal dermoid cysts constitute $1 \%-3 \%$ of all dermoids and $3.7 \%-12.6 \%$ of dermoids in the head and neck [2]. The other differential diagnosis of a midline nasal mass includes inflammatory lesions, post-traumatic deformities, benign and malignant neoplasms, developmental masses and vascular malformations.

Dermoid cysts contain ectodermal and mesodermal elements, including adnexal structures and sebaceous glands [3]. In contrast to teratomas, they contain no endodermal derived structures. Midline Nasal dermoids may present as a nasal mass, pit or fistula located anywhere from the glabella to columella. The majority of lesions are superficial with no deep extension; a variable reported 
percentage might end blindly within the deeper midline structures of the nose or extend intracranially [1]. They may secrete sebaceous material and can become intermittently inflamed or infected. Sequelae of infection can result in osteomyelitis, meningitis or cerebral abscess. These complications may result from inadequate surgical excision [4].

\section{Case Report}

A 15-year-old male came to our hospital with complaints of nasal pyramid deformity, widening of the dorsum of nose since childhood and nose blocks since one year. There was no history of nasal discharge/bleeding, frequent colds, excessive sneezing. There was a history of surgery for cyst over the dorsum of the nose when he was a few months old. We didn't find any other associated congenital anomalies.

On examination, there was swelling of the dorsum of nose associated with splaying of nasal bones. There was also Scar seen over the dorsum of the nose. The rest of the ENT examination was normal.

We ordered a CT scan of the nose and paranasal sinuses [Figure 1] which showed a swelling on the dorsum of the nose with splaying of the nasal bones. Also a MRI with contrast [Figure 2] was done which showed mixed signal intensity mass lesion which approximately measures $2.69 \times 1.50 \mathrm{cms}$ in the anterior nasal cavity within the anterior septum, associated with sinus tract extending superiorly to the foramen caecum with no intracranial extension.

Based on the history, clinical examination and radiological tests diagnosis of recurrent nasal dermoid was made.

After detailed evaluation, patient posted for recurrent nasal dermoid excision via external approach with scar revision and external rhinoplasty.

Under general anesthesia, infiltration of the dorsum of the nose was done with 1 in 100,000 lignocaine solution. Navigation protocol [Figure 3] is used because we were operating in critical area like skull base \& Dura.
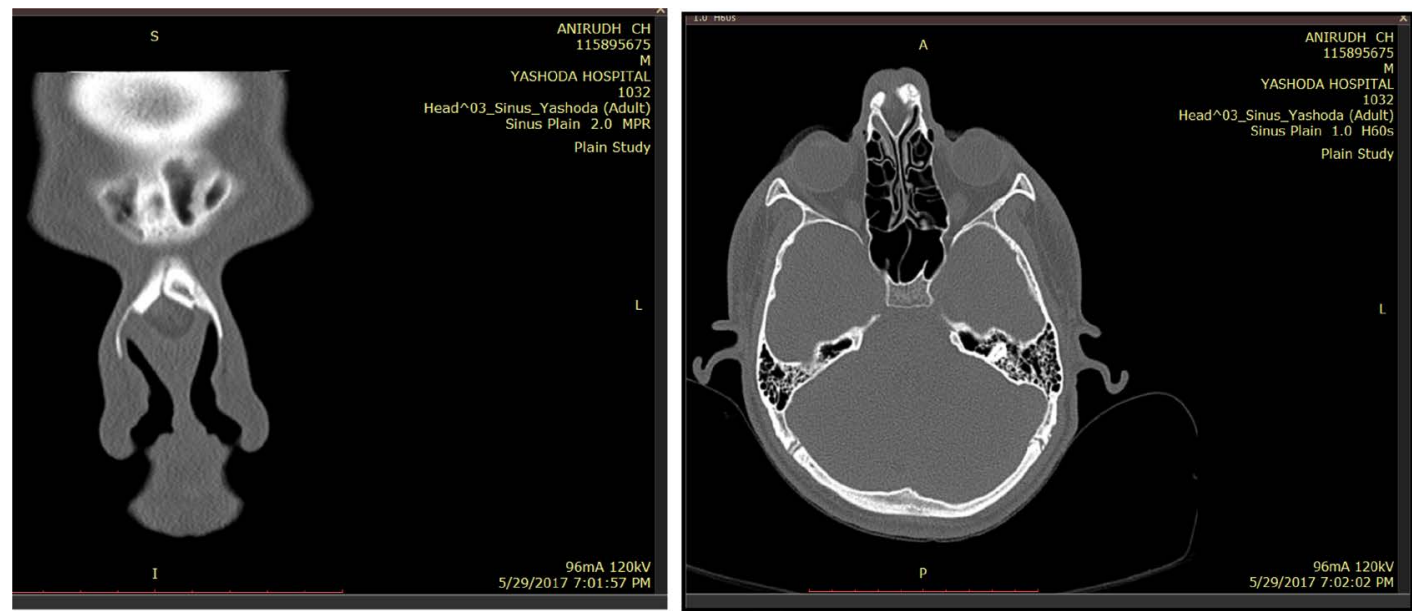

Figure 1. Computed tomography of nose and paranasal sinuses showed swelling over the dorsum of the nose with splaying of nasal bones. 


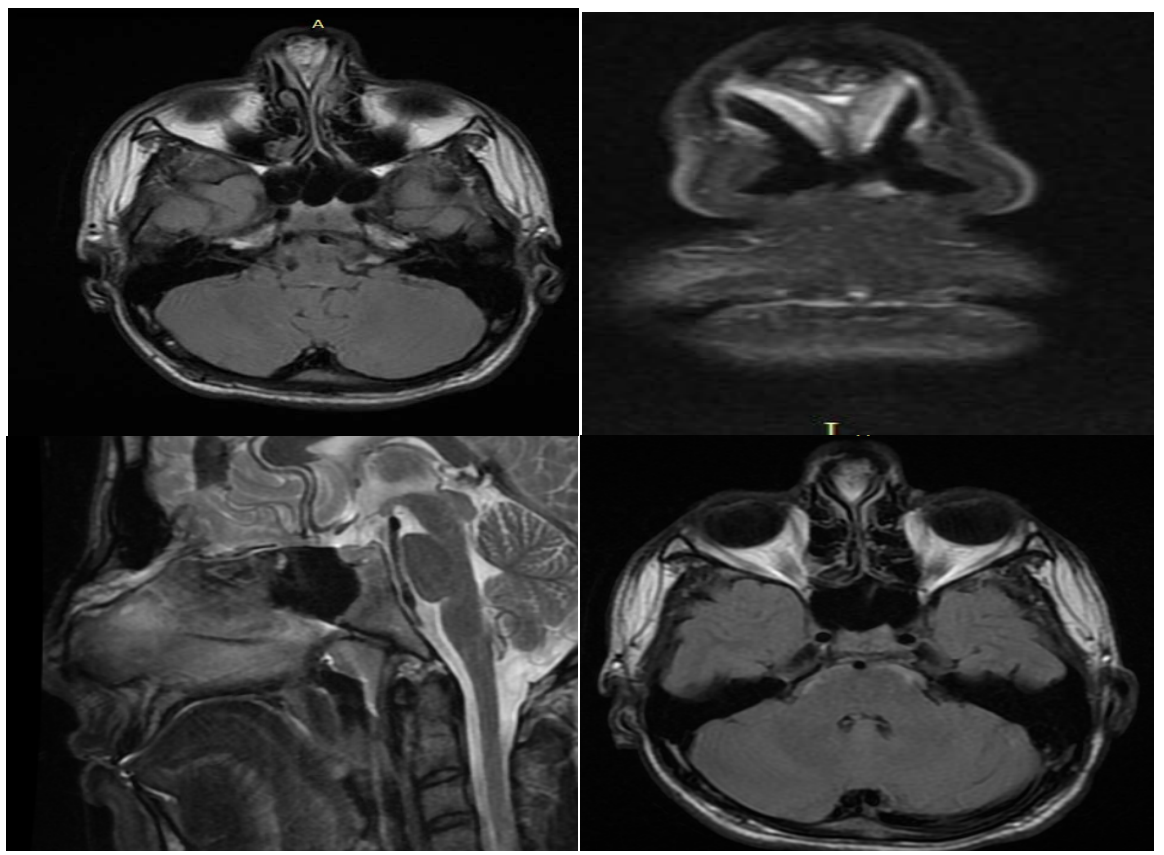

Figure 2. Magnetic resonance imaging of brain with gadolinium contrast showed mixed signal intensity mass lesion which approximately measures $2.69 \times 1.50 \mathrm{cms}$ in the anterior nasal cavity within the anterior septum, associated with sinus tract extending superiorly to the foramen caecum with no intracranial extension.
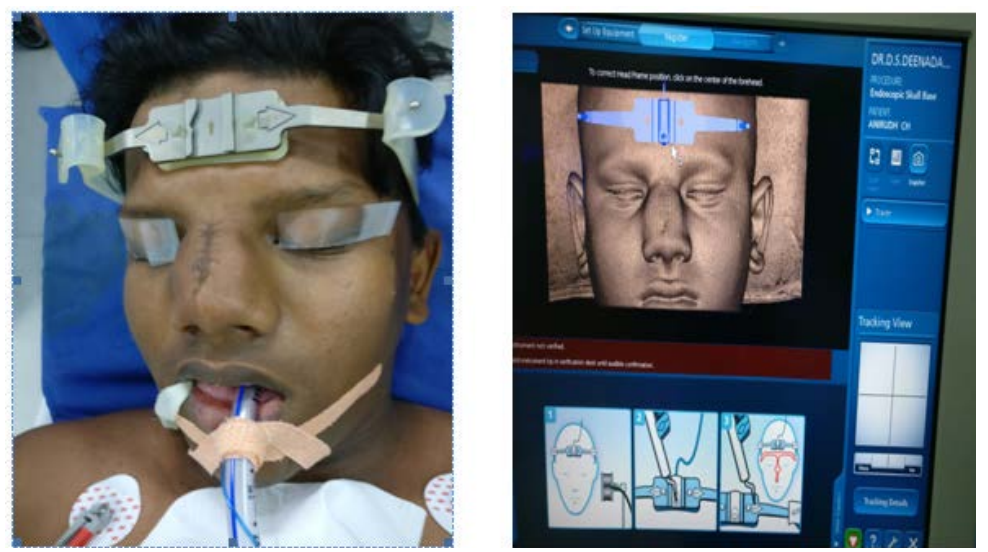

Figure 3. Navigation protocol.

\section{Surgical Procedure}

The elongated oval incision [Figure 4] was given over dorsum of the nose which includes the scar tissue. The fistula was seen over inferior part of the scar; dye injected into the fistula opening to delineate tract. The track was followed superiorly \& dissected all along from the floor of frontal sinus towards foramen caecum. Midline \& lateral osteotomies were done, nasal bones were approximated. Dead space was filled with harvested abdominal fat to achieve cosmoses. A nasal splint was kept.

The histopathology showing sinus tract is shown in Figure 5.

Pre and post operative comparative pictures are shown in Figure 6. 


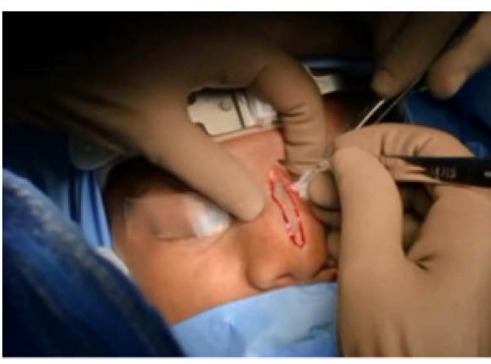

ELONGATED OVAL INCISION GIVEN ALONG WITH SCAR TISSUE

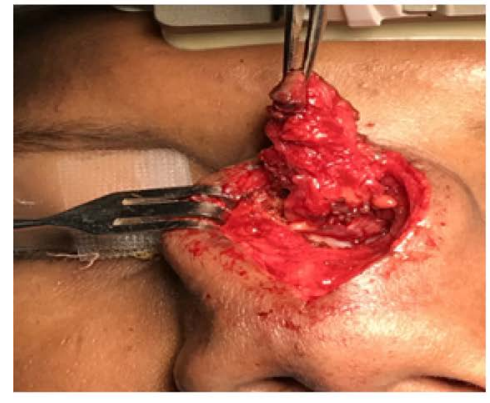

DISSECTED ALL ALONG

Figure 4. Showing steps of surgery.

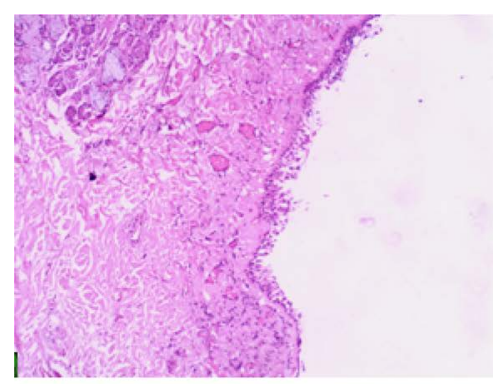

HISTOPATHOLOGY

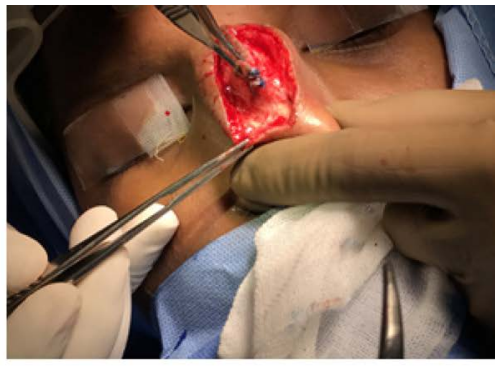

DYE INJECTED INTO SINUS OPENING

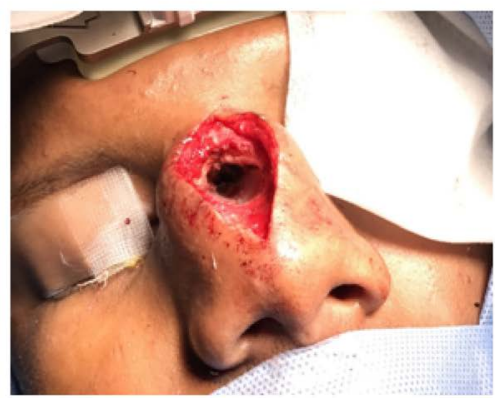

REMOVED INTOTO

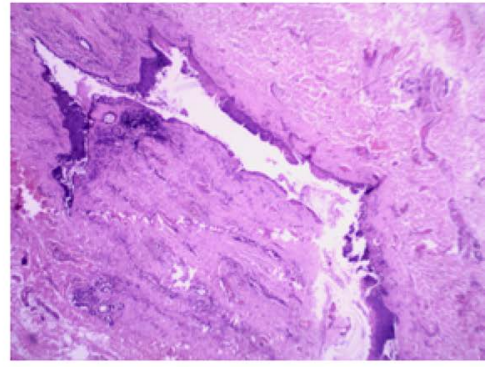

SINUS TRACT SEEN

Figure 5. Histopathology.

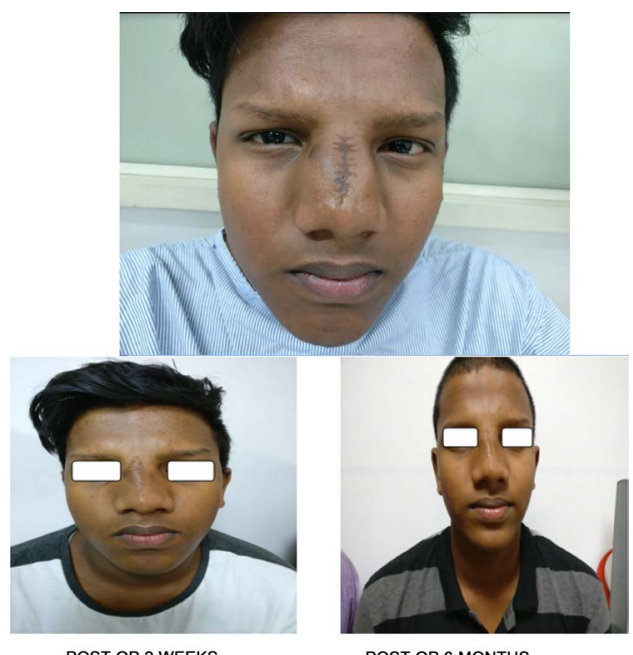

POST OP 2 WEEKS

POST OP 6 MONTHS

Figure 6. Pre operative and post operative comparative picture. 


\section{Discussion}

Nasal dermoid is the most common congenital midline nasal masses. This condition is usually diagnosed in children before the age of 3 years and very rarely later. They present mainly as a draining sinus or a pit or a deformity of the nose. Nasal dermoid is distinct from other facial dermoids in their potential to involve deeper contagious structures as well as intracranial spread. It is not uncommon for these cysts to have an intracranial extension hence a preoperative CT scan and an MRI is mandatory while evaluating these patients. A study by Winterton showed that the imaging studies also are unable to diagnose intracranial extension with good accuracy. They found positive and negative predictive values for intracranial extension were $85.7 \%$ and $50 \%$ for CT scan and were $100 \%$ and $50.0 \%$ for MRI [5].

An intraoperative extension of the sinus tract intracranial is conclusive and hence a provision for craniotomy if required should always be kept in mind while performing surgery. The treatment for nasal dermoid is complete surgical excision. Various approaches are used depending on the lesion and extension of the sinus tract ranging from local excision to a combined intracranial and extracranial approach. Emel Cadali used an open rhinoplasty approach in their patients. The reasons for them to choose this technique are exposure, good esthetic results and allow the reconstruction of the nasal dorsum [6].

In a study, Winterton reported 19 patients with nasal dermoid sinus cyst. Only one patient required local excision while the rest 18 patients underwent Bicoronal approach and amongst these 18 patients, 13 patients required craniotomy to excise the intracranial extension. They used a Bicoronal approach to facilitate craniotomy when needed intraoperative because imaging is unable to diagnose intracranial extension with sufficient accuracy [5]. We used a local excision technique with navigation in our patient along with medial and lateral osteotomies and augmented the nasal dorsum with fat to have an excellent cosmetic outcome.

The ideal surgical approach for extensive dermoid cysts and tracts should permit

- Access to the whole cyst/tract

- Medial and lateral osteotomies if required

- Rapid repair of cribriform defects and CSF rhinorrhea

- Reconstruction of the nasal dorsum with a minimally visible scar [7]

The recurrence rate of the nasal dermoid is about 20 percent, and Hamza et al. advocate the use of microscope or endoscope as an aid while excising these lesions [8].

Incomplete removal is the major reason for the recurrence.

We used navigation while performing this surgery that helped not just complete removal of the cyst but also helps avoid complication like damage to the skull base. No reports have been described so far where navigation has been used for removal of a dermoid. We recommend the usage of navigation for safety 
while performing the surgery.

\section{Conclusion}

Nasal dermoid cysts are rare entities. Preoperative workup with CT scan and MRI is required to exclude any intracranial extension. Complete surgical excision is the treatment of choice.

\section{References}

[1] Hughes, G.B., Sharpino, G., Hunt, W. and Tucker, H.M. (2018) Management of the Congenital Midline Nasal Mass: A Review. Head \& Neck Surgery, 2, 222-233. https://doi.org/10.1002/hed.2890020308

[2] Wardinsky, T.D., Pagon, R.A., Kropp, R.J., Hayden, P.W. and Clarren, S.K. (1991) Nasal Dermoid Sinus Cysts: Association with Intracranial Extension and Multiple Malformations. The Cleft Palate-Craniofacial Journal, 28, 87-95. https://doi.org/10.1597/1545-1569(1991)028<0087:NDSCAW >2.3.CO;2

[3] Kopeć, T., Wierzbicka, M. and Szyfter, W. (2013) Stensen's Duct Injuries: The Role of Sialendoscopy and Adjuvant Botulinum Toxin Injection. Wideochir Inne Tech Maloinwazyjne, 8, 112-116. https://doi.org/10.5114/wiitm.2011.32851

[4] Yavuzer, R., Bier, U. and Jackson, I.T. (1999) Be Careful: It Might Be a Nasal Dermoid Cyst. Plastic and Reconstructive Surgery, 103, 2082-2083.

https://doi.org/10.1097/00006534-199906000-00054

[5] Winterton, R.I.S., Wilks, D.J., Chumas, P.D., Russell, J.L. and Liddington, M.I. (2010) Surgical Correction of Midline Nasal Dermoid Sinus Cysts. Journal of Craniofacial Surgery, 21, 295-300. https://doi.org/10.1097/SCS.0b013e3181cf5f44

[6] Tatar, E.C., Selçuk, O.T., Saylam, G., Ozdek, A. and Korkmaz, H. (2009) The Management of Rare Nasal Mass-Nasal Dermoid Sinus Cysts: Open Rhinoplasty. Rare Tumors, 1, e40.

[7] Al-Reefy, H., Hopkins, C. and Jonas, N. (2018) Open Access Atlas of Otolaryngology, Head \& Neck Operative Surgery.

https://vula.uct.ac.za/access/content/group/ba5fb1bd-be95-48e5-81be-586fbaeba29d /Surgery for nasal dermoids.pdf

[8] Yıldız, H., Cil, Y., Simsek, H.A. and Erginay, T. (2011) Recurrent Adult Nasal Dermoid Cyst. Journal of Cutaneous and Aesthetic Surgery, 4, 151-152.

https://doi.org/10.4103/0974-2077.85046 